\title{
A SUCCESSFUL VOLUNTEER PROGRAM SHOWCASED DURING THE INTERNATIONAL YEAR OF VOLUNTEERING:THE CANCER COUNCIL NSW'S BREAST CANCER SUPPORT SERVICE
}

\section{Louise Burton}

The Cancer Council NSW

\section{Nicole Rankin}

National Breast Cancer Centre

\section{Afaf Girgis and Ann Weeden}

The Cancer Council NSW

How often do we read in or hear through the media the story of survivors, often referred to as heroes of events or incidents? These survivors portray courage, inner strength, resilience, and many other admirable qualities. Consider the influence of these portrayals on our own lives. We can apply a similar context to cancer survivors.

The Cancer Council NSW has long recognised the value of survivors who, in the role of trained volunteers, can show the importance of 'having been there'. Today, due to the advances in cancer treatments, more cancer patients will survive their experience of cancer. In Australia, the five-year relative survival ratio for all cancers is now 57 per cent in females and 46 per cent in males. ${ }^{1}$ If cancer survivors are well recruited, trained, and supported, they can act as mentors for newlydiagnosed cancer patients and their carers. This article describes an evaluation of the volunteer program of The Cancer Council NSW's Breast Cancer Support Service (BCSS).

\section{BACKGROUND}

The BCSS harnasses the positive experiences and recovery of volunteers to assist people newly diagnosed with breast cancer. The BCSS has been operating for over 25 years with great success. For many years the BCSS was entirely a volunteer run program; however, since the 1980s The Cancer Council NSW has provided funding for the infrastructure.

The BCSS is a major cancer support volunteer program, with more than 400 volunteers in NSW. The volunteers include women and men located throughout rural and urban areas. The service aims to ensure that volunteers are matched to new referrals as closely as possible by age, treatment type, social setting, and culture where applicable. The Cancer Council NSW strongly supports the view that the role of the volunteer cannot be replaced by a health professional. Unlike volunteers, health professionals have not experienced a diagnosis of cancer, its treatment, or its survival.

The BCSS relies on trained volunteers who have had a diagnosis of breast cancer. Survivors must be two years post-diagnosis and considered suitable by their treating specialist to apply. The selection process involves a formal panel interview, followed by an intensive training program over two days. At the end of training, it is decided whether applicants are suitable to become volunteers. The first few visits for a volunteer are closely supported by a coordinator, who also debriefs the volunteer. New volunteers also have a follow-up meeting with other volunteers six weeks into their role.

\section{STATEWIDE EVALUATION}

During 1998-2000, the Cancer Education Research Program of The Cancer Council NSW conducted a statewide evaluation of the service. ${ }^{2-5}$ The evaluation included surveys of volunteers, service coordinators, consumers, breast surgeons, and other health professionals from NSW public and private hospitals. Here, we focus exclusively on the volunteer and coordinator components.

The aims of the volunteer and coordinator components were to examine the levels of satisfaction among these groups, particularly in relation to their roles and responsibilities, and to ascertain their opinions of how the service might be improved and further promoted. Telephone interviews were conducted with 287 volunteers and 21 coordinators ( 85 per cent and 88 per cent of all eligible to participate, respectively). Most volunteers had visited at least 10 women in the 12 months preceding the interview (68 per cent) and reported that the majority of visits took place in hospitals (64 per cent) after women had had surgery (83 per cent).

\section{Volunteer responses}

Almost all volunteers agreed that most visits they made were positive and rewarding (98 per cent), and that they were able to help women by sharing experiences (96 per cent). Qualitative data collected in the interviews indicated that many volunteers gained particular satisfaction in being able to 'give something back' after their own experience of breast cancer and felt it was rewarding to let other women know they were not alone. Some volunteers commented about the need for further training, as many women asked for information about a broad range of issues. These diverse issues included breast reconstruction, spiritual issues, relaxation techniques, accessing prostheses and special clothing, and dealing with husbands and partners. Volunteers also commented about the need to promote the service ('We need to encourage early referral and promote it to all members of the medical community'); and about how to improve the service ('The service needs more volunteers, especially from different backgrounds').

\section{Coordinator responses}

Coordinators expressed high levels of satisfaction in dealing with volunteers and felt supported in their role by The Cancer Council NSW. Coordinators reported that 
it was easy to match new referrals and volunteers by characteristics such as age (79 per cent). However, it was sometimes difficult to match by characteristics such as personal circumstances such as having young children and by similar treatment types ( 39 per cent and 42 per cent, respectively). This was especially the case for coordinators in rural areas, where the number of volunteers on whom they could call was limited. With regards to improving the service, several coordinators commented about how they had designed better systems to ensure women were told about the BCSS: 'I'm going to develop a protocol with the local nursing unit manager, so there's a clear procedure for ringing the service whenever a new patient is diagnosed'. Qualitative data also revealed the specific issues that coordinators faced in promoting the service, particularly to women from rural areas: 'Many women are electing to have a mastectomy because they can't afford to be away from home for six weeks and have the radiotherapy during that time. But women aren't getting referred back to services in their own home towns. No one is there for them'.

\section{Implementation of recommendations}

Currently strategies are being developed to implement the recommendations made as a result of the evaluation. Recently, The Cancer Council NSW has been able to expand the service across NSW, through partnerships at the area health service level. This partnership has enabled the program to expand its capacity across all rural areas, helping to ensure access and equity for all consumers. The Cancer Council NSW is indebted to this continued collaboration with those area health services that have agreed that the BCSS program should be part of the role of senior health professionals in their areas.

The Cancer Council NSW has had the commitment of area health services in the following regions: the North West, Central West, Mid West, North Coast, Mid North Coast, Central Coast, Illawarra, South Coast, Southern and Great Murray. The commitment to providing the coordination in each area is a vital component to the program's success. The backgrounds of the 18 coordination staff include women's health coordinators, palliative care coordinators, Breastscreen nurse counsellors, stomal therapists, oncology nurse consultants, and cancer care coordinators.

\section{New challenges}

With the changing environment of breast cancer care, the BCSS faces numerous challenges. First, many women are now being discharged from hospital less than 48 hours after surgery. ${ }^{6}$ Consequently, it is often difficult to match a referral in a timely fashion that allows a woman to sufficiently recover from her surgery and be psychologically ready to meet a volunteer. In past years, many volunteers were not participating in the workforce and were more readily available to make visits. However, the evaluation reported that more than 50 per cent of volunteers were engaged in full-time or part-time employment. As a result, more careful planning is required in the referral process.

A second challenge for the service is the availability and role of the breast care nurse. Many major cancer care centres across NSW are now employing either specialist breast nurses or part-time breast nurses. The Cancer Council NSW views this as an opportunity to enhance the services offered to those people diagnosed with breast cancer. Recently, the Cancer Council NSW conducted some interviews with breast care nurses operating in the Sydney metropolitan area. This identified many opportunities to work together, in areas such as the training of new BCSS volunteers and in updating programs. This will allow a greater understanding of both the roles of the service and the breast care nurse and may open opportunities for further collaboration. For the person diagnosed with breast cancer, the future appears much brighter with the expansion of support in this area.

The Cancer Council NSW will continue to encourage the role of volunteers in its support programs. The model used by the BCSS has been translated into programs for people diagnosed with prostate and colorectal cancer. A pilot colorectal program is in operation at Nepean Hospital, which was initiated at the request of a patient in consultation with her surgeon. This program is currently being evaluated. The Cancer Council NSW is keen to explore further how the volunteer model can be successfully implemented in the case of other cancers and will continue to seek support for volunteer programs by developing and fostering partnerships within the current health system.

\section{REFERENCES}

1. Australian Institute of Health and Welfare. Australia's Health 2000: the seventh biennial health report of the Australian Institute of Health and Welfare. Canberra: AIHW, 2000.

2. Rankin N. Accessing and participating in psychosocial care: Australian women with breast cancer 2000-doctoral thesis, University of Newcastle, New South Wales, Australia (unpublished).

3. Rankin N, Girgis A. Evaluation of the NSW Cancer Council's Breast Cancer Support Service: Service Providers. Newcastle: New South Wales Cancer Council Cancer Education Research Program. 1999.

4. Rankin N, Girgis A. Evaluation of the NSW Cancer Council's Breast Cancer Support Service: Service Referrers. Newcastle: New South Wales Cancer Council Cancer Education Research Program. 1999.

5. Rankin N, Girgis A. Evaluation of the NSW Cancer Council's Breast Cancer Support Service: Service Consumers (Stage 1 report). Newcastle: New South Wales Cancer Council Cancer Education Research Program. 1999.

6. Davis C, Williams P, Redman S. Early discharge following breast surgery: assessing care, support, and informational needs of women with early breast cancer in Australia. Australian and New Zealand Journal of Surgery 2000; 70(8): 569-72. Fit 